\title{
Association of 25 hydroxyvitamin D concentration with risk of COVID-19: a Mendelian randomization study
}

Di Liu ${ }^{1 \#}$, Qiuyue Tian ${ }^{1 \#}$, Jie Zhang ${ }^{1}$, Haifeng $\mathrm{Hou}^{2}$, Wei Wang ${ }^{1,2,3}$, Qun Meng ${ }^{1}$, Youxin Wang ${ }^{1,2,4}$

${ }^{1}$ Beijing Key Laboratory of Clinical Epidemiology, School of Public Health, Capital Medical University, Beijing, 100069, China;

${ }^{2}$ School of Public Health, Shandong First Medical University \& Shandong Academy of Medical Sciences, Tai'an 271016, Shandong Province, China.

${ }^{3}$ School of Medical and Health Sciences, Edith Cowan University, Perth 60127, Australia

${ }^{4}$ Inner Mongolia Comprehensive Center for Disease Control and Prevention, Hohhot, Inner Mongolia Autonomous Region, China.

\#These authors contributed equally to this study.

Youxin Wang, PhD, Professor

School of Public Health, Capital Medical University

No.10 Xitoutiao, Youanmenwai Street, Fengtai District, Beijing, 100069, China

Tel: +8613581719105; wangy@ ccmu.edu.cn 


\section{Abstract}

Background In observational studies, 25 hydroxyvitamin D (25OHD) concentration has been associated with an increased risk of Coronavirus disease 2019 (COVID-19). However, it remains unclear whether this association is causal.

Methods We performed a two-sample Mendelian randomization (MR) to explore the causal relationship between $25 \mathrm{OHD}$ concentration and COVID-19, using summary data from the genome-wide association studies (GWASs) and using 25OHD concentration-related SNPs as instrumental variables (IVs).

Results MR analysis did not show any evidence of a causal association of 25OHD concentration with COVID-19 susceptibility and severity (odds ratio $[\mathrm{OR}]=1.136,95 \%$ confidence interval [CI] $0.988-1.306, P=0.074 ; \mathrm{OR}=0.889,95 \%$ CI $0.549-1.439$, $P=0.632$ ). Sensitivity analyses using different instruments and statistical models yielded similar findings, suggesting the robustness of the causal association. No obvious pleiotropy bias and heterogeneity were observed.

Conclusion The MR analysis showed that there might be no linear causal relationship of 25OHD concentration with COVID-19 susceptibility and severity.

Key words: Coronavirus disease 2019; vitamin D deficiency; 25 hydroxyvitamin D; Mendelian randomization 


\section{Introduction}

Coronavirus disease 2019 (COVID-19) has become a global pandemic ${ }^{1}$. Aimed at delaying disease onset by modulating modifiable risk factors, primary prevention has been proposed as a potentially effective and feasible tool to address the global challenge posed by COVID-19.

Vitamin $\mathrm{D}$ is a hormone, produced in the skin during exposure to sunlight, helped regulate the amount of calcium and phosphate in the body, which are needed to keep bones, teeth, and muscles healthy, and played a critical role in the immune system ${ }^{2-4}$. Vitamin D deficiency is a candidate risk factor for a range of adverse health outcomes, such as cancer, cardiovascular diseases, metabolic disorders, infectious diseases, as well as autoimmune diseases ${ }^{5,6}$. There were well recognized evidences that vitamin $\mathrm{D}$ deficiency contributes to the seasonal increase of virus infections of the respiratory tract, from the common cold to influenza, and now possibly also COVID-19 ${ }^{7,8}$. Some systematic reviews indicated that vitamin D deficiency may increase infection risk of COVID-19 by discussing the biological mechanism of virus infection ${ }^{4,9,10}$. In addition, numerous population-based studies have evaluated the Vitamin D deficiency in COVID-19 patients relative to controls; however, the findings have been inconsistent. Some studies showed that COVID-19 patients had a lower 25 hydroxyvitamin D (25OHD) concentration compared with healthy controls ${ }^{7,11-13}$, while others found no association ${ }^{14-16}$. The inconsistent findings from these epidemiological studies may be due to differences in the study 
design, study population, assays used for measuring Vitamin D deficiency. It is important to note that as the nature of the above studies is observational, such traditional epidemiological studies are particularly vulnerable to reverse causality and residual confounding.

A promising approach known as Mendelian randomization (MR), which uses inherited genetic variants as instrumental variables, provides stronger evidence for the causal effect of exposure on the diseases largely overcoming the traditional limitations due to confounding and reverse causality ${ }^{17,18}$. Summary based MR is an excellent strategy to evaluate the causality using summary statistics from Genome Wide Association Study (GWAS) data ${ }^{19,20}$. Therefore, we conducted MR analysis to assess whether 25OHD concentration was causally associated with risk of COVID-19.

\section{Methods}

\section{Data sources}

Genetic association datasets for COVID-19 susceptibility and severity

The summarized data was obtained from the most recent version of GWAS analyses from the COVID-19 host genetics initiative from UK Biobank individuals, which released on July 1, 2020 (https://www.covid19hg.org/results/) ${ }^{21}$. Summarized data on COVID-19 included two population, COVID-19 (n=6,696) vs. general population without the phenotype $(n=1073072)$ and COVID-19 (n=3,523) vs. COVID-19 negative $(n=36,634)$; and summarized data on severe COVID-19 included 536 patients and 329391 control participants from general population without the 
phenotype. In addition, we used summarized data for severe respiratory confirmed COVID-19 reported by from the Severe Covid-19 GWAS Group ${ }^{22}$. The GWAS summarized data can be downloaded at https://ikmb.shinyapps.io/COVID-19_GWAS_Browser/. The study included 1,160 patients who had severe respiratory confirmed COVID-19, and 2,205 participants from the general population without COVID-19 as the control.

Selection of $250 H D$ concentration -associated single nucleotide polymorphisms $(S N P S)$

We selected 143 SNPs as instrumental variables (IVs) for 25OHD concentration based on the recent large-scale GWAS ${ }^{23}$. In addition, we retained independent variants from each other (Linkage disequilibrium $[\mathrm{LD}], \mathrm{r}^{2}<0.001$ ) for sensitivity analysis. When we encountered genetic variants in LD, the SNP with the lowest $p$-value was selected. The LD proxies were defined using 1000 genomes European samples.

\section{MR analysis}

In the analyses, the inverse-variance weighted (IVW) method was used to estimate the overall causal association of 25OHD concentration on COVID-19 susceptibility and severity. We additionally conducted the weighted median, penalized weighted median, and MR-Egger regression to account for potential violations of valid instrumental variable assumptions. The MR-Egger analysis was performed to evaluate pleiotropy 
based on the intercept. We conducted a heterogeneity test in MR analyses using the IVW Q test. Then, sensitivity analyses were performed to examine the stability of the causal estimate. Firstly, we performed a "leave one out" analysis to further investigate the possibility that the causal association was driven by a single SNP. Then, we retained independent variants from each other $\left(\mathrm{LD}, \mathrm{r}^{2}<0.001\right)$ for further sensitivity analysis. Results were presented as odds ratios (OR) with their 95\% confidence interval (CI) and beta with standard error (SE) of outcomes per genetically predicted increase in each exposure factor.

In terms of various estimates for different measures, we chose the result of main MR method as the following rules:

(1) If no directional pleiotropy in MR estimates (Q statistic: $P$ value $>0.05$, MR-Egger intercept: $P$ value $>0.05)$, the results of the IVW method were reported.

(2) If directional pleiotropy was detected (MR-Egger intercept: $P$ value $<0.05$ ) and $P$ value $>0.05$ for the Q test, the results of the MR-Egger method were reported.

(3) If directional pleiotropy was detected (MR-Egger intercept: $P$ value $<0.05$ ) and $P$ value $<0.05$ for the $\mathrm{Q}$ test, the results of the weighted median method were reported.

All data analyses were performed by the "twosampleMR" package using $\mathrm{R}$ version 4.0.0 (https://www.r-project.org/).

\section{Results}

As shown in Table 1, the MR analysis showed no significant association of 
genetically instrumented 25OHD concentration with COVID-19 in the population of COVID-19 vs. population and COVID-19 vs. COVID-19 negative (OR=1.136, 95\% CI $0.988-1.306, P=0.074 ; \mathrm{OR}=1.168,95 \%$ CI $0.956-1.427, P=0.128)$. The association of 25OHD concentration with COVID-19 was robust in the weighted median and penalized weighted median methods, except in the MR-Egger regression $(\mathrm{OR}=1.258$, 95\% CI 1.053-1.502, $P=0.013 ; \mathrm{OR}=1.302,95 \%$ CI 1.011-1.676, $P=0.044)$. Pleiotropy bias and heterogeneity were also not observed. In terms of various estimates for different measures, we reported the results of the IVW method. In addition, the "leave one out" results showed that by omitting the included 89 SNPs one at a time, no individual genetic variants seem to have any significant effect on the overall results (Figure 1-2). The association of 25OHD concentration with COVID-19 remained robust using SNP instrument (LD, $\left.\mathrm{r}^{2}<0.001\right)$ (Table 1, Figure S1-2).

The MR analysis showed no significant association of genetically instrumented 25OHD concentration with severe COVID-19 in the population of severe respiratory confirmed COVID-19 vs. population reported by two groups $(\mathrm{OR}=0.889,95 \% \mathrm{CI}$ 0.549-1.439, $P=0.246 ; \mathrm{OR}=0.894,95 \%$ CI $0.587-1.363, P=0.603$ ) (Table 2). The result in the weighted median, penalized weighted median, and MR-Egger regression methods were robust. There was limited evidence of heterogeneity and horizontal pleiotropy based on the Q test and MR-Egger intercept test. Sensitivity analyses using different instruments yielded similar findings, suggesting the robustness of the causal association (Table 2, Figure 3-4, Figure S3-4). 


\section{Discussion}

In the current study, we performed an MR analysis to investigate the causal association of 25OHD concentration with the risk of COVID-19. Our results indicated that there might be no linear causal relationship of $250 \mathrm{HD}$ concentration with COVID-19 susceptibility and severity.

An observational study based on the UK Biobank data claimed that no link between vitamin D concentrations and risk of COVID-19 infection either overall or separated ethnic groups ${ }^{16}$. However, one study used causal inference analysis, supported the hypothesis that vitamin D plays a causal role in COVID-19 outcomes via modification of host responses to SARS-CoV-2 ${ }^{24}$. In addition, there were also systematic reviews and meta-analyses to explore the association between vitamin D and COVID-19 ${ }^{9,10,25}$. These studies provided a biological hypothesis and evolving epidemiological data supporting a role for vitamin D in COVID-19. But these results only based on the observational study design, which may be confounder bias. In our study, the SNPs associated with vitamin D as IVs were used to estimate the overall causal association of 25OHD concentration on COVID-19 susceptibility and severity, based on the MR design. The MR study could potentially avoid many biases and confounding issues existing in conventional observational studies and thus help to identify causally related risk factors. Using MR design, we found no evidence supporting that genetically predicted $25 \mathrm{OHD}$ concentration was significantly associated with COVID-19 susceptibility and severity. 
There are some possible explanations for these negative findings. First, these null findings suggest that the associations of $25 \mathrm{OHD}$ concentration with COVID-19 susceptibility and severity could attribute from the reverse causation bias and confounder bias. Vitamin D from environment with across to adequate sunshine or diet was metabolized in the liver to 25OHD, which was used to determine a patient's vitamin D status ${ }^{23,26}$. Vitamin D deficiency may be common in COVID-19 patients, as a consequence of quarantined and reduced outdoor behavior. The observed association between 25OHD concentration with increased risk of COVID-19 could be confounded by outdoor behavior which may be corrected with the genetic liability to COVID-19 ${ }^{23}$. Our findings suggested that COVID-19 susceptibility and severity are expected to decrease the prevalence of vitamin D deficiency, which are needed to be proved by more bi-directional MR studies. Second, it is also important to note that MR study considers the lifelong effect of genetic modification of COVID-19. However, the association between the vitamin D level and the risk of COVID-19 may be not fixed for a lifetime, but perform time-varying ${ }^{27}$. The cross-sectional observational nature of all current MR studies limits the evaluation. The future MR studies incorporating follow-up data should be considered the effect of vitamin D level on COVID-19 and how genetic variants effects change with time may impact the interpretability and validity of their results. Third, as shown by previous studies, vitamin D supplementation only shows treatment effects among individuals with baseline $25 \mathrm{OHD}$ concentration of no more than $30 \mathrm{nmol} / \mathrm{L}$, indicating that the relationship between the $25 \mathrm{OHD}$ concentration and the risk of diseases may be 
nonlinear ${ }^{28-30}$. However, we noted that there is a linearity assumption in our Mendelian randomization analyses ${ }^{23}$, then non-linear relationship could not be tested and might equate to the null hypothesis of no effect of the exposure on the outcome. Therefore, our results indicated that there might be no linear causal relationship of 25OHD concentration with COVID-19 susceptibility and severity.

The evidence of findings from MR studies sit at the interface between observational studies and RCTs ${ }^{31}$. RCTs provide interventions for disease, while MR studies could therefore not be directly extrapolated for this purpose, but could rather be used to provide evidence of a causal relationship. It should be also focused on the effect of vitamin D supplementation on COVID-19. The previous findings suggest that vitamin D deficiency and treatment has a long-term effect on preventing overall mortality ${ }^{12,32}$. In addition, future research should pay attention to not only the impact of vitamin D deficiency and treatment on the incidence of COVID-19, but also the impact of vitamin D deficiency and treatment on the COVID-19 mortality and lost life in COVID-19.

Some limitations should be noticed. It is important to note that the results of the MR analyses are based on numerous assumptions. First, we selected genetic variants as IVs based on the recent large-scale GWAS ${ }^{23}$, which showed a strong association with 25OHD concentration; therefore, the bias of weak instrument might be less likely. Second, the genetic variants are not associated with measured and unmeasured confounders that influence both vitamin D and COVID-19. However, the unmeasured confounders or alternative causal pathways may be still affected our results because of 
the limitation of the method. Third, the existence of horizontal pleiotropy may distort MR results. In our study, there was limited evidence of heterogeneity and horizontal pleiotropy. In addition, the GWAS of the severe COVID-19 cases included small sample size, which might lead to small effect for the MR estimate and limit the IVs for COVID-19 for reverse MR analysis. The findings were based on European population, which made it difficult to represent the universal conclusions for other ethnic groups. Therefore, the future studies with larger sample size and more ethnic groups are needed to verify and explore the observed associations.

\section{Conclusion}

Using 25OHD concentration-related SNPs as IVs from GWAS data, the MR analysis results indicated that there might be no linear causal relationship of 25OHD concentration with COVID-19 susceptibility and severity. In future, the bi-directional MR and non-linear MR study was needed to further prove these results. In addition, we should pay more attention to the randomization control trials about association between vitamin D treatment and the improvement of the COVID-19 in the long-term benefits.

\section{Availability of data and materials}

All data generated or analyzed during this study are included in this published article and its supplementary information files.

\section{Disclosure of Potential Conflicts of Interest}


All authors have approved the manuscript and its submission. No potential conflicts of interest were disclosed by the authors.

\section{Funding/Support}

The study was supported by grants from the National Natural Science Foundation of China (81872682 and 81773527), and the China-Australian Collaborative Grant (NSFC 81561128020-NHMRC APP1112767).

\section{Role of the Funder/Sponsor}

The funding organization had no role in the design and conduct of the study; collection, management, analysis, and interpretation of the data; preparation, review, or approval of the manuscript; or decision to submit the manuscript for publication. 


\section{References}

1. Thomson B. The COVID-19 Pandemic: A Global Natural Experiment. Circulation. 2020;142(1):14-16.

2. DeLuca HF. Vitamin D endocrinology. Annals of internal medicine. 1976;85(3):367-377.

3. Kulda V. [Vitamin D metabolism]. Vnitrni lekarstvi. 2012;58(5):400-404.

4. Aygun H. Vitamin D can prevent COVID-19 infection-induced multiple organ damage. Naunyn-Schmiedeberg's archives of pharmacology. 2020;393(7):1157-1160.

5. Holick MF, Chen TC. Vitamin D deficiency: a worldwide problem with health consequences. Am J Clin Nutr. 2008;87(4):1080S-1086S.

6. Hossein-nezhad A, Holick MF. Vitamin D for health: a global perspective. Mayo Clin Proc. 2013;88(7):720-755.

7. Meltzer DO, Best TJ, Zhang H, Vokes T, Arora V, Solway J. Association of Vitamin D Deficiency and Treatment with COVID-19 Incidence. medRxiv : the preprint server for health sciences. 2020.

8. Whittemore PB. COVID-19 fatalities, latitude, sunlight, and vitamin D. American journal of infection control. 2020.

9. Ghasemian R, Shamshirian A, Heydari K, et al. The Role of Vitamin D in The Age of COVID-19: A Systematic Review and Meta-Analysis Along with an Ecological Approach. medRxiv. 2020:2020.2006.2005.20123554.

10. Munshi R, Hussein MH, Toraih EA, et al. Vitamin D insufficiency as a potential culprit in critical COVID-19 patients. Journal of medical virology. 2020.

11. Merzon E, Tworowski D, Gorohovski A, et al. Low plasma 25(OH) vitamin D level is associated with increased risk of COVID-19 infection: an Israeli population-based study. The FEBS journal. 2020.

12. Pugach IZ, Pugach S. Strong Correlation Between Prevalence of Severe Vitamin D Deficiency and Population Mortality Rate from COVID-19 in Europe. medRxiv : the preprint server for health sciences. 2020:2020.2006.2024.20138644.

13. De Smet D, De Smet K, Herroelen P, Gryspeerdt S, Martens GA. Vitamin D deficiency as risk factor for severe COVID-19: a convergence of two pandemics. medRxiv : the preprint server for health sciences. 2020:2020.2005.2001.20079376.

14. Raisi-Estabragh Z, McCracken C, Bethell MS, et al. Greater risk of severe COVID-19 in Black, Asian and Minority Ethnic populations is not explained by cardiometabolic, socioeconomic or behavioural factors, or by $25(\mathrm{OH})$-vitamin D status: study of 1326 cases from the UK Biobank. Journal of public health (Oxford, England). 2020.

15. Panagiotou G, Tee SA, Ihsan Y, et al. Low serum 25-hydroxyvitamin D (25[OH]D) levels in patients hospitalised with COVID-19 are associated with greater disease severity. Clinical endocrinology. 2020.

16. Hastie CE, Mackay DF, Ho F, et al. Vitamin D concentrations and COVID-19 infection in UK Biobank. Diabetes and Metabolic Syndrome: Clinical Research and Reviews. 2020;14(4):561-565.

17. Davey Smith G, Hemani G. Mendelian randomization: genetic anchors for causal inference in epidemiological studies. Hum Mol Genet. 2014;23(R1):R89-98.

18. Sekula P, Del Greco MF, Pattaro C, Köttgen A. Mendelian Randomization as an Approach to 
Assess Causality Using Observational Data. Journal of the American Society of Nephrology : JASN. 2016;27(11):3253-3265.

19. Burgess S, Butterworth A, Thompson SG. Mendelian randomization analysis with multiple genetic variants using summarized data. Genet Epidemiol. 2013;37(7):658-665.

20. Dimou NL, Tsilidis KK. A Primer in Mendelian Randomization Methodology with a Focus on Utilizing Published Summary Association Data. Methods Mol Biol. 2018;1793:211-230.

21. Initiative C-HG. The COVID-19 Host Genetics Initiative, a global initiative to elucidate the role of host genetic factors in susceptibility and severity of the SARS-CoV-2 virus pandemic. European journal of human genetics : EJHG. 2020;28(6):715-718.

22. Ellinghaus D, Degenhardt F, Bujanda L, et al. Genomewide Association Study of Severe Covid-19 with Respiratory Failure. N Engl J Med. 2020.

23. Revez JA, Lin T, Qiao Z, et al. Genome-wide association study identifies 143 loci associated with 25 hydroxyvitamin D concentration. Nature communications. 2020;11(1):1647.

24. Davies G, Garami AR, Byers JC. Evidence Supports a Causal Role for Vitamin D Status in COVID-19 Outcomes. medRxiv : the preprint server for health sciences. 2020:2020.2005.2001.20087965.

25. Laird E, Rhodes J, Kenny RA. Vitamin D and inflammation: Potential implications for severity of Covid-19. Irish medical journal. 2020;113(5).

26. Holick MF. Vitamin D deficiency. The New England journal of medicine. 2007;357(3):266-281.

27. Meng JE, Hovey KM, Wactawski-Wende J, et al. Intraindividual variation in plasma 25-hydroxyvitamin D measures 5 years apart among postmenopausal women. Cancer epidemiology, biomarkers \& prevention : a publication of the American Association for Cancer Research, cosponsored by the American Society of Preventive Oncology. 2012;21(6):916-924.

28. Crowe FL, Thayakaran R, Gittoes N, et al. Non-linear associations of 25-hydroxyvitamin D concentrations with risk of cardiovascular disease and all-cause mortality: Results from The Health Improvement Network (THIN) database. The Journal of steroid biochemistry and molecular biology. 2019;195:105480.

29. Esteghamati A, Fotouhi A, Faghihi-Kashani S, et al. Non-linear contribution of serum vitamin D to symptomatic diabetic neuropathy: A case-control study. Diabetes research and clinical practice. 2016;111:44-50.

30. Zittermann A. Vitamin D Status, Supplementation and Cardiovascular Disease. Anticancer research. 2018;38(2):1179-1186.

31. Davies NM, Holmes MV, Smith GD. Reading Mendelian randomisation studies: a guide, glossary, and checklist for clinicians. Bmj Clinical Research. 2018;362:k601.

32. Ouchetto O, Drissi Bourhanbour A. Risk Factors for Mortality of COVID-19 Patients. medRxiv : the preprint server for health sciences. 2020:2020.2007.2002.20145375. 
Figure legends

Figure 1. MR leave-one-out sensitivity analysis for '25OHD concentration' on 'COVID-19' in the population of COVID-19 vs. population

Leave-one-out analysis: each row represents a MR analysis of 25OHD concentration on COVID-19 using all instruments expect for the SNP listed on the y-axis. The point represents the beta with that SNP removed and the line represents $95 \%$ confidence interval. The summary data are reported by the COVID-19 host genetics initiative.

COVID-19: Coronavirus disease 2019; MR: mendelian randomization; SNP, single-nucleotide polymorphism; 25OHD: 25-hydroxyitamin D; SD, standard deviation

Figure 2. MR leave-one-out sensitivity analysis for '25OHD concentration' on 'COVID-19' in the population of COVID-19 vs. COVID-19 negative

Leave-one-out analysis: each row represents a MR analysis of 25OHD concentration on COVID-19 using all instruments expect for the SNP listed on the y-axis. The point represents the beta with that SNP removed and the line represents $95 \%$ confidence interval. The summary data are reported by the COVID-19 host genetics initiative.

COVID-19: Coronavirus disease 2019; MR: mendelian randomization; SNP, single-nucleotide polymorphism; 25OHD: 25-hydroxyitamin D; SD, standard deviation 
Figure 3. MR leave-one-out sensitivity analysis for '25OHD concentration' on 'severe COVID-19' in the population of severe respiratory confirmed COVID-19 vs. population

Leave-one-out analysis: each row represents a MR analysis of 25OHD concentration on severe COVID-19 using all instruments expect for the SNP listed on the y-axis. The point represents the beta with that SNP removed and the line represents $95 \%$ confidence interval. The summary data are reported by the COVID-19 host genetics initiative.

COVID-19: Coronavirus disease 2019; MR: mendelian randomization; SNP, single-nucleotide polymorphism; 25OHD: 25-hydroxyitamin D; SD, standard deviation

Figure 4. MR leave-one-out sensitivity analysis for '25OHD concentration' on 'severe COVID-19' in the population of severe respiratory confirmed COVID-19

\section{vs. population}

Leave-one-out analysis: each row represents a MR analysis of 25OHD concentration on severe COVID-19 using all instruments expect for the SNP listed on the y-axis. The point represents the beta with that SNP removed and the line represents $95 \%$ confidence interval. The summary data are reported by the severe COVID-19 GWAS Group.

COVID-19: Coronavirus disease 2019; MR: mendelian randomization; SNP, single-nucleotide polymorphism; 25OHD: 25-hydroxyitamin D; SD, standard 
deviation; GWAS, genome wide association study

Figure S1. MR leave-one-out sensitivity analysis for '25OHD concentration' on 'COVID-19' in the population of COVID-19 vs. population

Leave-one-out analysis: each row represents a MR analysis of 25OHD concentration on COVID-19 using all instruments expect for the SNP listed on the y-axis. The point represents the beta with that SNP removed and the line represents $95 \%$ confidence interval. The summary data are reported by the COVID-19 host genetics initiative.

COVID-19: Coronavirus disease 2019; MR: mendelian randomization; SNP, single-nucleotide polymorphism; 25OHD: 25-hydroxyitamin D; SD, standard deviation

Figure S2. MR leave-one-out sensitivity analysis for '25OHD concentration' on 'COVID-19' in the population of COVID-19 vs. COVID-19 negative

Leave-one-out analysis: each row represents a MR analysis of 25OHD concentration on COVID-19 using all instruments expect for the SNP listed on the y-axis. The point represents the beta with that SNP removed and the line represents $95 \%$ confidence interval. The summary data are reported by the COVID-19 host genetics initiative.

COVID-19: Coronavirus disease 2019; MR: mendelian randomization; SNP, single-nucleotide polymorphism; 25OHD: 25-hydroxyitamin D; SD, standard deviation 
Figure S3. MR leave-one-out sensitivity analysis for '25OHD concentration' on 'severe COVID-19' in the population of severe respiratory confirmed COVID-19

\section{vs. population}

Leave-one-out analysis: each row represents a MR analysis of 25OHD concentration on severe COVID-19 using all instruments expect for the SNP listed on the y-axis. The point represents the beta with that SNP removed and the line represents $95 \%$ confidence interval. The summary data are reported by the COVID-19 host genetics initiative.

COVID-19: Coronavirus disease 2019; MR: mendelian randomization; SNP, single-nucleotide polymorphism; 25OHD: 25-hydroxyitamin D; SD, standard deviation

Figure S4. MR leave-one-out sensitivity analysis for '25OHD concentration' on 'severe COVID-19' in the population of severe respiratory confirmed COVID-19

\section{vs. population}

Leave-one-out analysis: each row represents a MR analysis of 25OHD concentration on severe COVID-19 using all instruments expect for the SNP listed on the y-axis. The point represents the beta with that SNP removed and the line represents $95 \%$ confidence interval. The summary data are reported by the severe COVID-19 GWAS Group.

COVID-19: Coronavirus disease 2019; MR: mendelian randomization; SNP, single-nucleotide polymorphism; 25OHD: 25-hydroxyitamin D; SD, standard 
medRxiv preprint doi: https://doi.org/10.1101/2020.08.09.20171280; this version posted August 18, 2020. The copyright holder for this preprint (which was not certified by peer review) is the author/funder, who has granted medRxiv a license to display the preprint in perpetuity.

All rights reserved. No reuse allowed without permission.

deviation; GWAS, genome wide association study 
Table 1 Causal association of 25OHD concentration with COVID-19

\begin{tabular}{|c|c|c|c|c|c|c|c|c|}
\hline Method & $\mathrm{SNP}(\mathrm{n})$ & OR $(95 \% \mathrm{CI})$ & Beta (SE) & $P$ & $\mathrm{SNP}(\mathrm{n})$ & OR $(95 \% \mathrm{CI})$ & Beta (SE) & $P$ \\
\hline \multicolumn{9}{|c|}{ COVID-19 vs. general population } \\
\hline IVW & 89 & $1.136(0.988-1.306)$ & $0.128(0.071)$ & 0.074 & 56 & $1.048(0.882-1.245)$ & $0.047(0.088)$ & 0.596 \\
\hline Weighted median & 89 & $1.155(0.936-1.425)$ & $0.144(0.107)$ & 0.179 & 56 & $1.142(0.915-1.426)$ & $0.133(0.113)$ & 0.239 \\
\hline Penalised weighted median & 89 & $1.155(0.939-1.420)$ & $0.144(0.106)$ & 0.173 & 56 & $1.142(0.913-1.429)$ & $0.133(0.114)$ & 0.244 \\
\hline MR_Egger & 89 & $1.258(1.053-1.502)$ & $0.229(0.090)$ & 0.013 & 56 & $1.149(0.918-1.439)$ & $0.139(0.115)$ & 0.230 \\
\hline$\beta$ (intercept) & 89 & - & $-0.007(0.004)$ & 0.071 & 56 & - & $-0.006(0.005)$ & 0.214 \\
\hline Q statistic & 89 & - & - & 0.759 & 56 & - & - & 0.794 \\
\hline \multicolumn{9}{|c|}{ COVID-19 vs. COVID-19 negative } \\
\hline IVW & 89 & $1.168(0.956-1.427)$ & $0.156(0.102)$ & 0.128 & 57 & $1.192(0.945-1.504)$ & $0.176(0.118)$ & 0.138 \\
\hline Weighted median & 89 & $1.017(0.750-1.379)$ & $0.017(0.156)$ & 0.912 & 57 & $1.134(0.810-1.588)$ & $0.126(0.172)$ & 0.464 \\
\hline Penalised weighted median & 89 & $0.990(0.737-1.328)$ & $-0.010(0.150)$ & 0.945 & 57 & $1.134(0.814-1.579)$ & $0.126(0.169)$ & 0.4570 . \\
\hline MR_Egger & 89 & $1.302(1.011-1.676)$ & $0.26(0.13)$ & 0.044 & 57 & $1.265(0.951-1.682)$ & $0.235(0.145)$ & 0.112 \\
\hline$\beta$ (intercept) & 89 & - & $-0.007(0.005)$ & 0.174 & 57 & - & $-0.004(0.006)$ & 0.485 \\
\hline Q statistic & 89 & - & - & 0.656 & 57 & - & - & 0.925 \\
\hline
\end{tabular}

The summary data are reported by the COVID-19 host genetics initiative.

Beta is the estimated effect size.

25OHD: 25 hydroxyvitamin D; CI: confidence intervals; COVID-19: Coronavirus disease 2019; IVs: instrumental variables; IVW, inverse-variance weighted; MR mendelian randomization; OR: odds ratio; SE, standard error; SNP, single-nucleotide polymorphism. 
Table 2 Causal association of $250 H D$ concentration with severe COVID-19

\begin{tabular}{|c|c|c|c|c|c|c|c|c|}
\hline Method & $\mathrm{SNP}(\mathrm{n})$ & OR $(95 \% \mathrm{CI})$ & Beta (SE) & $P$ & $\mathrm{SNP}(\mathrm{n})$ & OR $(95 \% \mathrm{CI})$ & Beta (SE) & $P$ \\
\hline \multicolumn{9}{|c|}{ Severe respiratory confirmed COVID-19 vs. general population } \\
\hline IVW & 82 & $0.889(0.549-1.439)$ & $-0.118(0.246)$ & 0.632 & 55 & $0.936(0.532-1.647)$ & $-0.067(0.288)$ & 0.818 \\
\hline Weighted median & 82 & $1.242(0.618-2.498)$ & $0.217(0.356)$ & 0.543 & 55 & $1.294(0.605-2.767)$ & $0.257(0.388)$ & 0.507 \\
\hline Penalised weighted median & 82 & $1.241(0.620-2.482)$ & $0.216(0.354)$ & 0.542 & 55 & $1.293(0.585-2.857)$ & $0.257(0.405)$ & 0.526 \\
\hline MR_Egger & 82 & $0.944(0.509-1.749)$ & $-0.058(0.315)$ & 0.855 & 55 & $0.998(0.477)$ & $-0.002(0.376)$ & 0.996 \\
\hline$\beta$ (intercept) & 82 & - & $-0.004(0.012)$ & 0.760 & 55 & - & $-0.004(0.015)$ & 0.790 \\
\hline Q statistic & 82 & - & - & 0.793 & 55 & - & - & 0.650 \\
\hline \multicolumn{9}{|c|}{ Severe respiratory confirmed COVID-19 vs. general population* } \\
\hline IVW & 82 & $0.894(0.587-1.363)$ & $-0.112(0.215)$ & 0.603 & 53 & $0.780(0.467-1.300)$ & $-0.249(0.261)$ & 0.340 \\
\hline Weighted median & 82 & $0.638(0.335-1.215)$ & $-0.450(0.329)$ & 0.171 & 53 & $0.633(0.322-1.244)$ & $-0.458(0.345)$ & 0.185 \\
\hline Penalised weighted median & 82 & $0.636(0.339-1.195)$ & $-0.452(0.321)$ & 0.160 & 53 & $0.633(0.318-1.261)$ & $-0.457(0.352)$ & 0.193 \\
\hline MR_Egger & 82 & $0.894(0.523-1.527)$ & $-0.112(0.273)$ & 0.682 & 53 & $0.711(0.365-1.387)$ & $-0.341(0.341)$ & 0.322 \\
\hline$\beta$ (intercept) & 82 & - & $0.00003(0.011)$ & 0.997 & 53 & - & $0.006(0.014)$ & 0.678 \\
\hline Q statistic & 82 & - & - & 0.789 & 53 & - & - & 0.555 \\
\hline
\end{tabular}

The summary data are reported by the COVID-19 host genetics initiative.

* The summary data are reported by the Severe Covid-19 GWAS Group.

Beta is the estimated effect size.

25OHD: 25 hydroxyvitamin D; CI: confidence intervals; COVID-19: Coronavirus disease 2019; IVs: instrumental variables; IVW, inverse-variance weighted; MR mendelian randomization; OR: odds ratio; SE, standard error; SNP, single-nucleotide polymorphism. 
rs12794714

rs12056768edRxiv preprint doi: https://doi-org/10.1101/2020.08.09.20171280; this version posted August 18, 2020. The copyright holder for-this preprint rs6782190 rs6671730

rs61891388

rs727857

rs2037511

rs11264322

rs72834856

rs35408430

rs2847500

rs590215

rs11542462

rs 1047891

rs8113404

rs12317268

rs17216707

rs11182428

rs7569755

rs13104260

rs8091117

rs10908419

rs2725371

rs12123821

rs4418728

rs10887718

rs11458206

rs4327060

rs11108368

rs77924615

rs2346264

rs62007299

rs2952289

rs138385079

rs9861009

rs11127186

rs7604788

rs115621755

rs1260326

rs61883501

rs28364331

rs13284054

rs142158911

rs4616820

rs3925446

rs1933064

rs9490317

rs200210321

rs4575545

rs325384

rs28374650

rs705117

rs2207132

rs2762943

rs10454087

rs212100

rs10642047

rs11076175

rs1038165

rs11732896

rs 10426

rs112943319

rs7149014

rs78151190

rs10908465

rs6123359

rs10085881

rs77532868

rs1660839

rs2012736

rs117576073

rs1352846

rs4364259

rs187429064

rs541041

rs55829990

rs7412

rs73413596

rs2131925

rs31612

rs140371183

rs12803256

rs613808

rs116970203

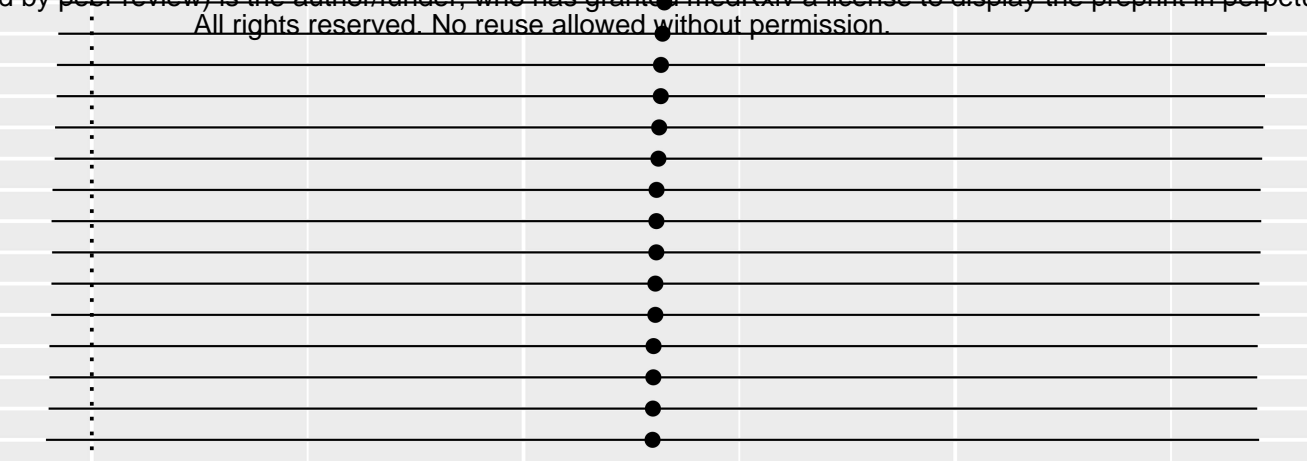


rs10642047 rs6188350 rs13284054 rs7149014 rs17216707 rs2207132 rs2847500 rs325384 rs1933064 rs72834856 rs61891388 rs77532868 rs10887718

rs10085881 rs212100

rs4616820

rs11732896 rs1038165 rs3925446 rs9861009 s2952289 rs73413596 rs28374650 rs31612 rs28364331 rs1047891 rs117576073 rs187429064 rs1260326 rs6123359

rs112943319

rs1212382

rs10908465

rs78151190

rs4364259 rs10426 rs7412 rs142158911 rs2012736 rs140371183 rs1660839 rs2131925 rs12803256 rs613808 rs116970203

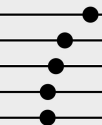


rs12056768 rs8091117

rs117576073

rs13104260

rs72997623

rs2952289

rs4575545

rs13284054

rs1149605

rs12317268

rs112943319

rs138385079

rs11542462

rs3925446

rs2762943

rs4327060

rs4364259

rs11076175

rs55829990

rs11264322 rs541041

rs10908419

rs7604788

rs6782190

rs73413596

rs8113404

rs61883501

rs77924615

rs10908465

rs7569755

rs2725371

rs6671730

rs62007299

rs7149014

rs35408430

rs11108368

rs11127186

rs10454087

rs11182428

rs10887718

rs705117

rs4616820

rs78151190

rs1038165

rs613808

rs7528419

rs11458206

rs9490317

rs590215

rs325384

rs4418728

rs6123359

rs77532868

rs11732896

rs 10085881

rs1933064

rs72834856

rs727857

rs2037511

rs31612

rs1660839

rs10642047

rs2346264

rs2207132

rs2012736

rs12803256

rs12798050

rs1352846

Al 
rs6123339 GRxiv preprint doi: https://doi org/10 1101/2020 080920171280 ; this version posted August 18, 2020 The copyright holder for this preprin rs2207132

rs10426

rs11076175

rs8091117

rs2037511

rs7149014

rs9490317

rs11182428

rs138385079

rs12056768

rs62007299

rs142158911

rs72834856

rs112943319

rs727857

rs 1260326

rs7569755

rs72997623

rs10908419

rs2952289

rs325384

rs11542462

rs13104260

rs3925446

rs1038165

rs7604788

rs11458206

rs11108368

rs7412

rs2725371

rs2847500

rs55829990

rs6671730

rs11264322

rs61883501

rs73413596

rs35408430

rs77532868

rs12317268

rs 4418728

rs6782190

rs1933064

rs77924615

rs10908465

rs10642047

rs10887718

rs10085881

rs78151190

rs1047891

rs4364259

rs11127186

rs116970203

rs2762943

rs590215

rs13284054

rs9861009

rs2131925

rs4327060

rs17216707

rs613808

rs8113404

rs2346264

rs705117

rs61891388

rs541041

rs 4616820

rs 4575545

rs 10454087

rs11732896

rs1149605

rs7528419

rs10859995

rs 212100

rs12794714

rs12798050

(which was not certified by peer review) is the author/funder, who has granted medRxiv a license to display the preprint in perpetuity.

All rights reserved. No reuse allowed without permission.

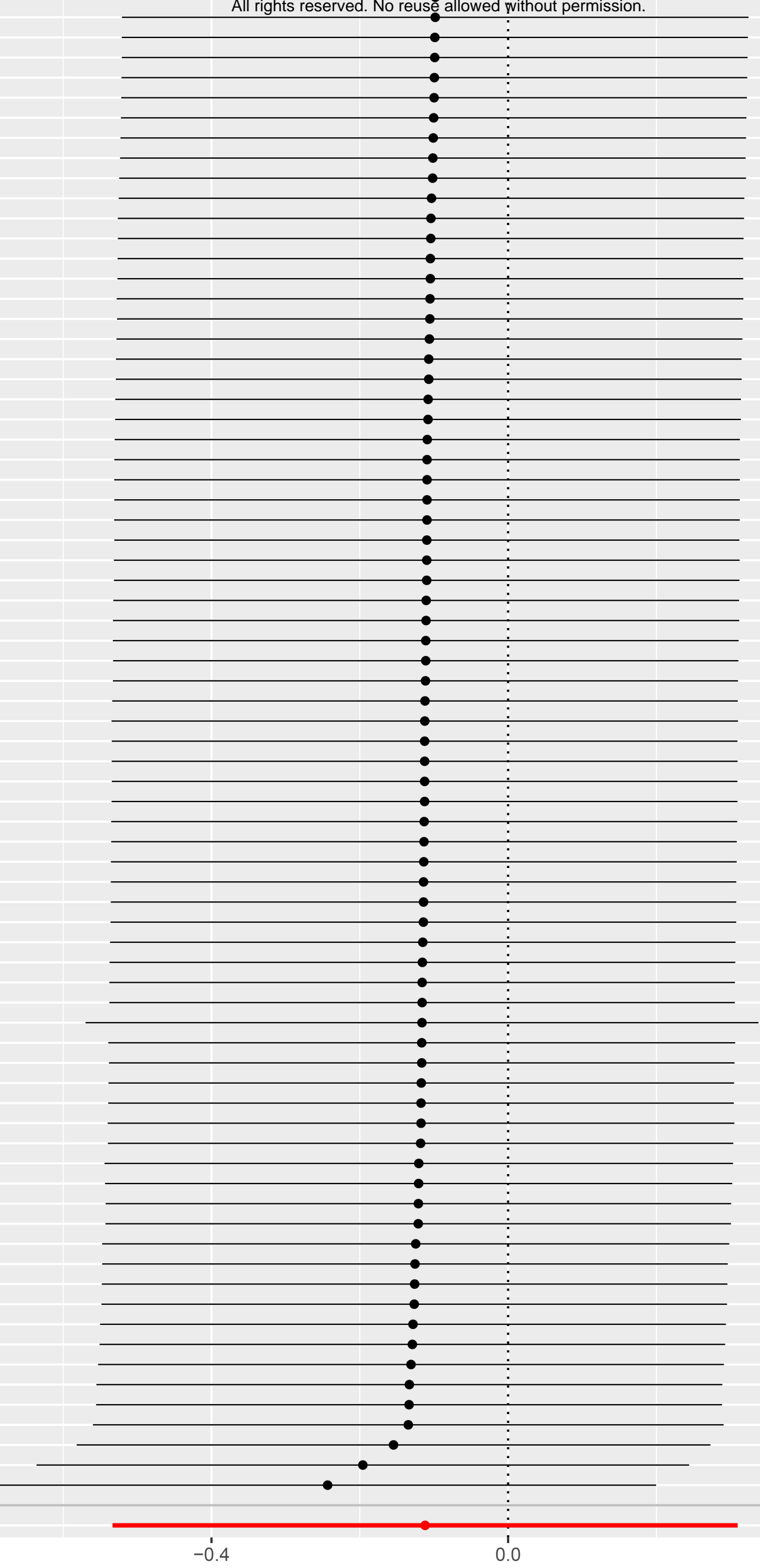

Beta of severe COVID-19 per SD of $250 \mathrm{HD}$ concentration 